\title{
Wide Range Image-shift Functions in SEM for Probing Applications
}

\author{
T. Agemura, ${ }^{*}$ M. Sato, ${ }^{*}$ T. Mizuno, ${ }^{* *}$ H. Yanagita, ${ }^{* *}$ and F. Yano** \\ * Beam Technology Center, R\&D Division, Nanotechnology Products Business Group, Hitachi High- \\ Technologies Corporation, Hitachinaka, Ibaraki 312-8504, Japan \\ ** Wafer Process Engineering Development Division, Renesas Technology Corporation, Kodaira, Tokyo \\ 187-8588, Japan
}

We report here on mage-shift functions introduced to the newly developed probing system, Hitachi N6000 Nano-Prober, which allow us to move a primary electron beam more than $200 \mu \mathrm{m}$ area. The NanoProber can analyze electrical characteristics of real transistors by using more than four probes touched directly to contacts or lines of LSI devices. Operators manually control the probes and confirm target plugs with which the probes will be in contact, as observing SEM images. In case that many probes should be in contact with the plugs, it is inevitable to move a view area of SEM image with a magnification that the contacts between the probes and the plugs can be recognized, because all plugs aren't always observed in the same view area. For moving the view area, moving a specimen stage mechanically or moving the primary electron beam electrically (image-shift) are available. Since the stage movement usually has some mechanical vibrations, the contacted probes can be destroyed. The image-shift has not only no mechanical vibration but the faster and more accurate movement than the stage. Meanwhile, the image-shift cannot perfectly cover the movable area of the stage, but it is important to realize an image-shift area of more than $200 \mu \mathrm{m}$ in view of the memory mat size or the arrangement of plugs related to gates or substrates of current semiconductor devices.

While the image-shift is operated, the primary beam is incident into an objective lens at some angles, which deteriorates the resolution of SEM images due to off-axis aberrations [1], as shown in FIG.1. However, those aberrations can be mitigated by controlling an incident position of the primary beam into the objective lens (an optical axis condition) in response to the amount of image-shift movement. Consequently, alignment signals were dynamically superimposed on image-shift signals in order to control the optical axis conditions. We could hereby obtain desired resolution for probing applications of $15 \mathrm{~nm}$ at the working distance of 15 $\mathrm{mm}$ and the accelerating voltage of $5 \mathrm{kV}$ within all over the square area of $240 \mu \mathrm{m}$.

In case that the optical axis conditions are dynamically controlled in accordance with the amount of image shift movement, an irradiating position of the primary beam is displaced to the other position on the specimen as shown in FIG. 2; therefore an image-shift function is required that the primary beam must be accurately shifted to desired positions on the specimen. The amount of the image-shift movement, or imageshift coordinates $W_{I S}$ on the specimen (complex number) is given by,

$$
W_{I S}=A \cdot w_{I S}
$$

where, $w_{I S}$ is the controlled variable of the image-shift coils (complex number), and $A$ is the coefficient (complex number) determined by electron optics conditions, an arrangement of aligners, rotational angle of the primary beam due to the objective lens, and so on. The image-shift coils were controlled as calculating $A$ based on the operational conditions of the electron optical system, and the accuracy of less than $2 \%$ was realized for each image-shift movement in the Nano-Prober in the conditions of the working distance of 15 $\mathrm{mm}$, the accelerating voltage of $5 \mathrm{kV}$, and the fixed crossover of the condenser lens. We have also 
developed the functions for indicating the image-shift coordinates on the SEM screen, which is very useful for the Nano-Prober in case of complexly patterned specimens.

Finally, we have also theoretically considered how to realize an achromatic axis condition of the objective lens for low accelerating voltage applications, and how to prevent the displacement of the image-shift coordinates for more accurate control of image-shift movement.

\section{References}

[1] P. W. Hawkes et al., Principles of electron optics, Academic Press, London, 1996.

[2] The Authors would like to thank Mrs. O. Yamada, H. Sato and M. Watahiki of Hitachi Science Systems Corporation for their tremendous help on this work.

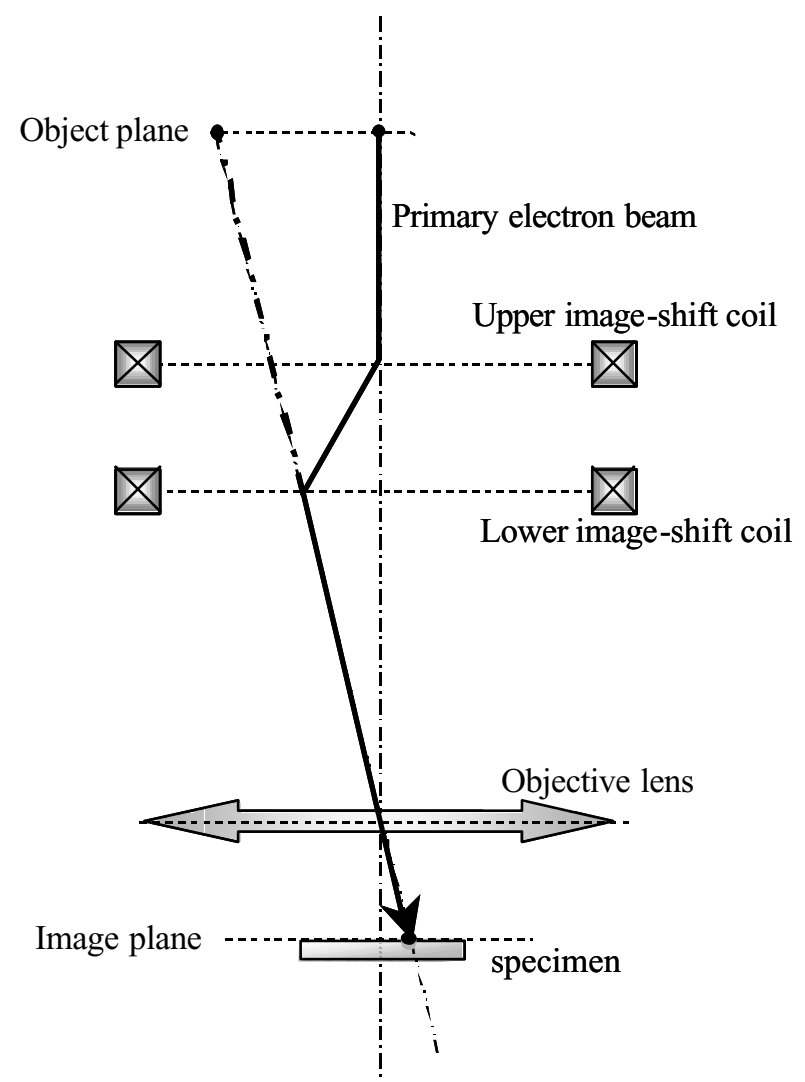

FIG. 1. Principle for shifting the primary electron beam on the specimen by image-shift coils. The primary beam is incident into the objective lens at some angle.

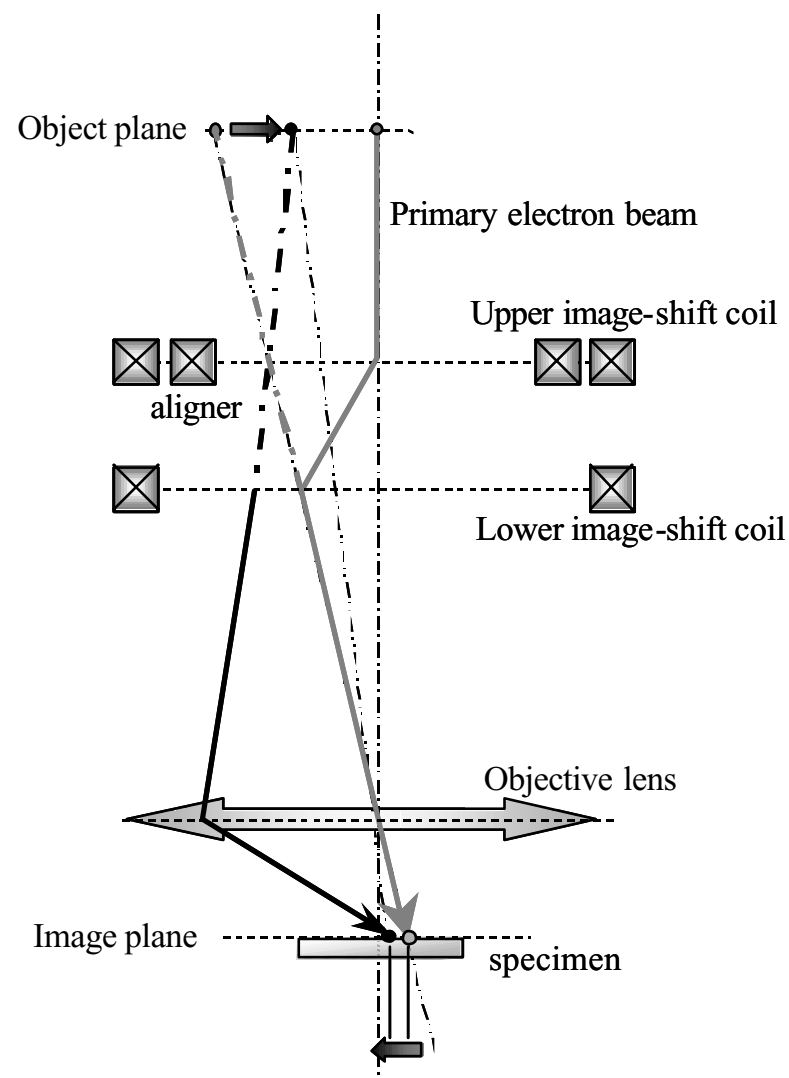

FIG. 2. Displacement of the primary beam on the specimen when the aligner is running for controlling the optical axis. 